\title{
HOMOGENEOUS LENGTH FUNCTIONS ON GROUPS
}

\author{
D.H.J. POLYMATH
}

\begin{abstract}
A pseudo-length function defined on an arbitrary group $G=\left(G, \cdot, e,()^{-1}\right)$ is a map $\ell: G \rightarrow[0,+\infty)$ obeying $\ell(e)=0$, the symmetry property $\ell\left(x^{-1}\right)=\ell(x)$, and the triangle inequality $\ell(x y) \leqslant$ $\ell(x)+\ell(y)$ for all $x, y \in G$. We consider pseudo-length functions which saturate the triangle inequality whenever $x=y$, or equivalently those that are homogeneous in the sense that $\ell\left(x^{n}\right)=n \ell(x)$ for all $n \in \mathbb{N}$. We show that this implies that $\ell([x, y])=0$ for all $x, y \in G$. This leads to a classification of such pseudo-length functions as pullbacks from embeddings into a Banach space. We also obtain a quantitative version of our main result which allows for defects in the triangle inequality or the homogeneity property.
\end{abstract}

\section{INTRODUCTION}

Let $G=\left(G, \cdot e,()^{-1}\right)$ be a group (written multiplicatively, with identity element $e$ ). A pseudo-length function on $G$ is a map $\ell: G \rightarrow[0,+\infty)$ that obeys the properties

- $\ell(e)=0$,

- $\ell\left(x^{-1}\right)=\ell(x)$,

- $\ell(x y) \leqslant \ell(x)+\ell(y)$

for all $x, y \in G$. If in addition we have $\ell(x)>0$ for all $x \in G \backslash\{e\}$, we say that $\ell$ is a length function. By setting $d(x, y):=\ell\left(x^{-1} y\right)$, it is easy to see that pseudo-length functions (resp. length functions) are in bijection with left-invariant pseudometrics (resp. left-invariant metrics) on $G$.

From the above properties it is clear that one has the upper bound

$$
\ell\left(x^{n}\right) \leqslant|n| \ell(x)
$$

for all $x \in G$ and $n \in \mathbb{Z}$. Let us say that a pseudo-length function $\ell: G \rightarrow$ $[0,+\infty)$ is homogeneous if equality is always attained here, in that one has

$$
\ell\left(x^{n}\right)=|n| \ell(x)
$$

for all $x \in G$ and any $n \in \mathbb{Z}$. Using the axioms of a pseudo-length function, it is not difficult to show that the homogeneity condition (1.1) is equivalent to the triangle inequality holding with equality whenever $x=y$ (i.e., that (1.1) holds for $n=2)$; see [7, Lemma 1].

If one has a real or complex Banach space $\mathbb{B}=(\mathbb{B},\|\|)$, and $\phi: G \rightarrow \mathbb{B}$ is any homomorphism from $G$ to $\mathbb{B}$ (viewing the latter as a group in additive notation), then the function $\ell: G \rightarrow[0,+\infty)$ defined by $\ell(x):=\|\phi(x)\|$ is easily verified to be a homogeneous pseudo-length function. Furthermore, if

2010 Mathematics Subject Classification. 20F12, 20F65.

Key words and phrases. homogeneous length function, pseudo-length function, quasimorphism, Banach space embedding. 
$\phi$ is injective, then $\ell$ is in fact a homogeneous length function. For instance, the function $\ell((n, m)):=|n+\sqrt{2} m|$ is a length function on $\mathbb{Z}^{2}$, where in this case $\mathbb{B}:=\mathbb{R}$ and $\phi((n, m)):=n+\sqrt{2} m$. On the other hand, one can easily locate many length functions that are not homogeneous, for instance by taking the square root of the length function just constructed.

The main result of this paper is that such Banach space constructions are in fact the only way to generate homogeneous (pseudo-)length functions.

Theorem 1.2 (Classification of homogeneous length functions). Given a group $G$, let $\ell: G \rightarrow[0,+\infty)$ be a homogeneous pseudo-length function. Then there exist a real Banach space $\mathbb{B}=(\mathbb{B},\|\|)$ and a group homomorphism $\phi: G \rightarrow \mathbb{B}$ such that $\ell(x)=\|\phi(x)\|$ for all $x \in G$. Furthermore, if $\ell$ is a length function, one can take $\phi$ to be injective, i.e., an isometric embedding.

We derive Theorem 1.2 from a more quantitative result bounding the pseudo-length of a commutator

$$
[x, y]:=x y x^{-1} y^{-1}
$$

see Proposition 2.1 below. Our arguments are elementary, relying on directly applying the axioms of a homogeneous length function to various carefully chosen words in $x$ and $y$, and repeatedly taking an asymptotic limit $n \rightarrow \infty$ to dispose of error terms that arise in the estimates obtained in this fashion.

An additional advantage of quantifying Theorem 1.2 in Proposition 2.1 is that one can derive from the latter proposition a "quasified" version of Theorem 1.2. See Theorem 4.4 below. ${ }^{1}$

Finally, as one quick corollary of Theorem 1.2, we obtain the following characterization of the groups that admit homogeneous length functions.

Corollary 1.4. A group admits a homogeneous length function if and only if it is abelian and torsion-free.

1.1. Examples and approaches. We now make some remarks to indicate the nontriviality of Theorem 1.2. Corollary 1.4 implies that there are no nonabelian groups with homogeneous length functions. Whether or not such a striking geometric rigidity phenomenon holds was previously unknown to experts. Moreover, the corollary fails to hold if one or more of the precise conditions in the theorem are weakened. For instance, such length functions indeed exist (i) on non-abelian monoids, and (ii) on balls of finite radius in free groups. We explain these two cases further in Section 4.

Given these cases, one could a priori ask if every non-abelian group admits a homogeneous length function. This is not hard to disprove; here are two examples.

Example 1.5 (Nilpotent groups). If $G$ is nilpotent of nilpotency class two (e.g., the Heisenberg group), then $[x, y]^{n^{2}}=\left[x^{n}, y^{n}\right]$ for all $x, y \in G$ and

\footnotetext{
${ }^{1} \mathrm{~A}$ different variant of Theorem 1.2 involves replacing homogeneity by the assumption that $\ell$ is a pseudo-length function on $G$ whose homogenization is positive:

$$
\ell_{\text {hom }}(g):=\lim _{n \rightarrow \infty} \frac{\ell\left(g^{n}\right)}{n}>0, \quad \forall g \neq e .
$$

(This was studied in $[15$, Theorem $2.10($ III)] in the special case of abelian $(G, \ell)$.) In this case we work with $\left(G, \ell_{\text {hom }}\right)$ instead of $\ell$, to conclude that $G$ maps into a Banach space.
} 
integers $n \geqslant 0$ since the map $(g, h) \mapsto[g, h]$ is now a bihomomorphism $G \times G \rightarrow[G, G] \subset Z(G)$. If $[x, y]$ is non-trivial, then any homogeneous length function on $G$ would assign a linearly growing quantity to the righthand side and a quadratically growing quantity to the left-hand side, which is absurd; thus such groups cannot admit homogeneous length functions. The claim then also follows for nilpotent groups of higher nilpotency class, since they contain subgroups of nilpotency class two. ${ }^{2}$

Example 1.6 (Connected Lie groups). As we explain in Remark 2.9, a homogeneous length function $\ell$ induces a bi-invariant metric on $G$. Now if $(G, \ell)$ is furthermore a connected Lie group, then by [13, Lemma 7.5], $G \cong K \times \mathbb{R}^{n}$ for some compact Lie group $K$ and integer $n \geqslant 0$. By (1.1), $K$ cannot have torsion elements, hence must be trivial. But then $G$ is abelian.

Prior to Corollary 1.4, the above examples left open the question of whether any non-abelian group admits a homogeneous length function. One may as well consider groups generated by two non-commuting elements. As a prototypical example, let $\mathbf{F}_{2}$ be the free group on two generators $a, b$. The word length function on $\mathbf{F}_{2}$ is a length function, but it is not homogeneous, since for instance the word length of $\left(b a b^{-1}\right)^{n}=b a^{n} b^{-1}$ is $n+2$, which is not a linear function of $n$. It is however the case that the word length of $x^{n}$ has linear growth in $n$ for any non-trivial $x$. Similarly for the Levenshtein distance (edit distance) on $\mathbf{F}_{2}$.

Our initial attempts to construct homogeneous length functions on $\mathbf{F}_{2}$ all failed. Of course, this failure is explained by our main result. However, many of these methods apply under minor weakening of the hypotheses, such as working with monoids rather than groups, or weakening homogeneity. Results in these cases are discussed further in Section 4.

1.2. Further motivations. We next mention some motivations from functional analysis and probability, or more precisely the study of Banach space embeddings. If $G$ is an additive subgroup of a Banach space $\mathbb{B}$, then clearly the norm on $\mathbb{B}$ restricts to a homogeneous length function on $G$. In $[4,7]$ one can find several equivalent conditions for a given length function on a given group to arise in this way (studied in the broader context of additive mappings and separation theorems in functional analysis); see also [15, Theorem 2.10(II)] for an alternative proof. These conditions are summarized in [10]. For instance, given a group $G$ with a length function $\ell$, there exists an isometric embedding from $G$ to a Banach space $\mathbb{B}$ with $\ell$ induced from the metric on $\mathbb{B}$, if and only if $G$ is amenable and $\ell\left(x^{2}\right)=2 \ell(x)$ for all $x$.

In view of such equivalences, it is natural to try and characterize the groups possessing a homogeneous length function. This question is answered by Corollary 1.4, which shows these are precisely the abelian torsion-free groups.

Groups and semigroups with translation-invariant metrics also naturally arise in probability theory, with the most important 'normed' (i.e., homogeneous) examples being Banach spaces [12]. Notice however that in certain

\footnotetext{
${ }^{2}$ One can also show by relatively simple means that solvable non-abelian groups cannot admit homogeneous length functions either; see the discussion on lamplighter groups in the comments to terrytao. wordpress. com/2017/12/16/.
} 
fundamental stochastic settings, formulating and proving results does not require the full Banach space structure. In this vein, a general variant of the Hoffmann-Jørgensen inequality was shown in [9] in arbitrary metric semigroups - including Banach spaces as well as (non-abelian) compact Lie groups. Similarly in [10], the authors transferred the (sharp) KhinchinKahane inequality from Banach spaces to abelian groups $G$ equipped with a homogeneous length function. To explore extensions of these results to the non-abelian setting (e.g., Lie groups with left-invariant metrics), we need to first understand if such objects exist. As explained above, this question was not answered in the literature; but it is now settled by our main result.

Finally, there may also be a relation to the Ribe program [14], which aims to reformulate aspects of Banach space theory in purely metric terms. Indeed, from Corollary 1.4 we see that a metric space $X$ is isometric to an additive subgroup of a Banach space if and only if there is a group structure on $X$ which makes the metric left-invariant and the length function $\ell(x):=d(1, x)$ homogeneous.

\section{ACKNOWLEDGEMENTS}

This project is an online collaboration that originated from a blog post at https://terrytao.wordpress . com/2017/12/16, following the model of the "Polymath" projects [8]. A full list of participants and their grant acknowledgments may be found at http://michaelnielsen.org/polymath1/index.php?title=linear_nor We also thank Michal Doucha for useful references and comments, in particular in bringing the paper [15] to our attention, and the anonymous referee for helpful suggestions.

\section{KEY PROPOSITION}

The key proposition used to prove Theorem 1.2 is the following estimate, which can treat a somewhat more general class of functions than homogeneous pseudo-length functions, in which the symmetry hypothesis is dropped and one allows for an error in the homogeneity property, which is now also only claimed for $n=2$.

Proposition 2.1. Let $G=(G, \cdot)$ be a group, let $c \in \mathbb{R}$, and let $\ell: G \rightarrow \mathbb{R}$ be a function obeying the following axioms:

(i) For any $x, y \in G$, one has

$$
\ell(x y) \leqslant \ell(x)+\ell(y) .
$$

(ii) For any $x \in G$, one has

$$
\ell\left(x^{2}\right) \geqslant 2 \ell(x)-c .
$$

Then for any $x, y \in G$, one has

$$
\ell([x, y]) \leqslant 5 c,
$$

where the commutator $[x, y]$ was defined in (1.3).

Notably, we neither assume symmetry $\ell\left(x^{-1}\right)=\ell(x)$, not even up to a constant, nor $\ell(e)=0$ (although $0 \leqslant \ell(e) \leqslant c$ follows from the axioms); we also allow $\ell$ to take on negative values. The reader may however wish to restrict attention to homogeneous length functions, and set $c=0$ and 
$\ell \geqslant 0$ for a first reading of the arguments below. The factor of 5 is probably not optimal here, but the crucial feature of the bound (2.4) for our main application is that the right-hand side vanishes when $c=0$ (the right-hand side is also independent of $x$ and $y$, which we use in other applications).

We define a semi-length function to be a function $\ell: G \rightarrow \mathbb{R}$ such that for all $x, y \in G, \ell(x y) \leqslant \ell(x)+\ell(y)$, i.e. $\ell$ satisfies (2.2). Every pseudo-length function is a semi-length function. A semi-length function that satisfies (2.3) for some $c \in \mathbb{R}$ is called quasi-homogeneous.

Remark 2.5. Suppose $\ell: G \rightarrow \mathbb{R}$ and there is a constant $k$ such that $\ell(x y) \leqslant \ell(x)+\ell(y)+k$ for all $x, y \in G$. Then the function $\ell^{\prime}(x):=\ell(x)+k$ is a semi-length function. Further, $\ell^{\prime}$ satisfies (2.3) with $c$ replaced by $c^{\prime}:=k+c$, whenever $\ell$ satisfies (2.3) on the nose. Thus Proposition 2.1 continues to hold if $(2.2)$ is replaced by the condition $\ell(x y) \leqslant \ell(x)+\ell(y)+k$ for all $x, y \in G$, with the bound in the conclusion (2.4) becoming $5 c+4 k$.

We now turn to the proof. For the remainder of this section, let $G, c$, and $\ell$ satisfy the hypotheses of the proposition. Our task is to establish the bound (2.4). We shall now use (2.2) and (2.3) repeatedly to establish a number of further inequalities relating the semi-lengths $\ell(x)$ of various elements $x$ of $G$, culminating in (2.4). Many of our inequalities will involve terms that depend on an auxiliary parameter $n$, but we will be able to eliminate several of them by the device of passing to the limit $n \rightarrow \infty$. It is because of this device that we are able to obtain a bound (2.4) whose right-hand side is completely uniform in $x$ and $y$.

From (2.2) and induction we have the upper homogeneity bound

$$
\ell\left(x^{n}\right) \leqslant n \ell(x)
$$

for any natural number $n \geqslant 1$. Similarly, from (2.3) and induction one has the lower homogeneity bound

$$
\ell\left(x^{n}\right) \geqslant n \ell(x)-\log _{2}(n) c \geqslant n \ell(x)-n c
$$

whenever $n$ is a power of two. It is convenient to rearrange this latter inequality as

$$
\ell(x) \leqslant \frac{\ell\left(x^{n}\right)}{n}+c .
$$

This inequality, particularly in the asymptotic limit $n \rightarrow \infty$, will be the principal means by which the hypothesis (2.3) is employed.

We remark that by further use of (2.6) one can also obtain a similar estimate to (2.7) for natural numbers $n$ that are not powers of two, but the powers of two will suffice for the arguments that follow.

Lemma 2.8 (Approximate conjugation invariance). For any $x, y \in G$, one has

$$
\ell\left(y x y^{-1}\right) \leqslant \ell(x)+c .
$$

Remark 2.9. Setting $c=0$, we conclude that any homogeneous pseudolength function is conjugation invariant, and thus determines a bi-invariant metric on $G$. It should not be surprising that this observation is used in the proof of Theorem 1.2, since it is a simple consequence of that theorem. 
Proof of Lemma 2.8. From (2.7) with $x$ replaced by $y x y^{-1}$, one has

$$
\ell\left(y x y^{-1}\right) \leqslant \frac{\ell\left(y x^{n} y^{-1}\right)}{n}+c
$$

whenever $n$ is a power of two. On the other hand, from (2.6) and (2.2) one has

$$
\ell\left(y x^{n} y^{-1}\right) \leqslant \ell(y)+n \ell(x)+\ell\left(y^{-1}\right)
$$

and thus

$$
\ell\left(y x y^{-1}\right) \leqslant \ell(x)+c+\frac{\ell(y)+\ell\left(y^{-1}\right)-c}{n} .
$$

Sending $n \rightarrow \infty$, we obtain the claim.

Lemma 2.10 (Splitting lemma). Let $x, y, z, w \in G$ be such that $x$ is conjugate to both wy and $z w^{-1}$. Then one has

$$
\ell(x) \leqslant \frac{\ell(y)+\ell(z)}{2}+\frac{3}{2} c .
$$

Proof. If we write $x=$ swys $^{-1}=t z w^{-1} t^{-1}$ for some $s, t \in G$, then from (2.7) we have

$$
\begin{aligned}
\ell(x) & \leqslant \frac{\ell\left(x^{n} x^{n}\right)}{2 n}+c \\
& =\frac{\ell\left(s(w y)^{n} s^{-1} t\left(z w^{-1}\right)^{n} t^{-1}\right)}{2 n}+c
\end{aligned}
$$

whenever $n$ is a power of two. From Lemma 2.8 and (2.2) one has

$$
\begin{aligned}
\ell\left((w y)^{k+1} s^{-1} t\left(z w^{-1}\right)^{k+1}\right) & =\ell\left(w y(w y)^{k} s^{-1} t\left(z w^{-1}\right)^{k} z w^{-1}\right) \\
& \leqslant \ell\left(y(w y)^{k} s^{-1} t\left(z w^{-1}\right)^{k} z\right)+c \\
& \leqslant \ell\left((w y)^{k} s^{-1} t\left(z w^{-1}\right)^{k}\right)+\ell(y)+\ell(z)+c
\end{aligned}
$$

for any $k \geqslant 0$, and hence by induction

$$
\ell\left((w y)^{n} s^{-1} t\left(z w^{-1}\right)^{n}\right) \leqslant \ell\left(s^{-1} t\right)+n(\ell(y)+\ell(z)+c) .
$$

Inserting this into the previous bound for $\ell(x)$ via two applications of $(2.2)$, we conclude that

$$
\ell(x) \leqslant \frac{\ell(y)+\ell(z)+c}{2}+\frac{\ell(s)+\ell\left(s^{-1} t\right)+\ell\left(t^{-1}\right)}{2 n}+c ;
$$

sending $n \rightarrow \infty$, we obtain the claim.

Corollary 2.12. If $x, y \in G$, let $f=f_{x, y}: \mathbb{Z}^{2} \rightarrow \mathbb{R}$ denote the function

$$
f(m, k):=\ell\left(x^{m}[x, y]^{k}\right) .
$$

Then for any $m, k \in \mathbb{Z}$, we have

$$
f(m, k) \leqslant \frac{f(m-1, k)+f(m+1, k-1)}{2}+2 c .
$$

Proof. Observe that $x^{m}[x, y]^{k}$ is conjugate to both $x\left(x^{m-1}[x, y]^{k}\right)$ and to $\left(y^{-1} x^{m}[x, y]^{k-1} x y\right) x^{-1}$, hence by $(2.11)$ one has

$$
\ell\left(x^{m}[x, y]^{k}\right) \leqslant \frac{\ell\left(x^{m-1}[x, y]^{k}\right)+\ell\left(y^{-1} x^{m}[x, y]^{k-1} x y\right)}{2}+\frac{3}{2} c .
$$


Since $y^{-1} x^{m}[x, y]^{k-1} x y$ is conjugate to $x^{m+1}[x, y]^{k-1}$, the claim now follows from Lemma 2.8.

We now prove Proposition 2.1. Let $x, y \in G$. We can write the inequality (2.13) in probabilistic form as

$$
f(m, k) \leqslant \mathbf{E} f\left(\left(m, k-\frac{1}{2}\right)+Y\left(1,-\frac{1}{2}\right)\right)+2 c
$$

where $Y= \pm 1$ is a Bernoulli random variable that equals 1 or -1 with equal probability. The key point here is the drift of $\left(0,-\frac{1}{2}\right)$ in the right-hand side. Iterating this inequality, we see that

$$
f(0, n) \leqslant \mathbf{E} f\left(\left(Y_{1}+\cdots+Y_{2 n}\right)\left(1,-\frac{1}{2}\right)\right)+4 c n,
$$

where $n \geqslant 0$ and $Y_{1}, \ldots, Y_{2 n}$ are independent copies of $Y$ (so in particular $Y_{1}+\cdots+Y_{2 n}$ is an even integer).

From (2.2) and (2.6) one has the inequality

$$
\begin{aligned}
f(m, k) \leqslant & |m|\left(\max \left(\ell(x), \ell\left(x^{-1}\right)\right)\right) \\
& +|k|\left(\max \left(\ell([x, y]), \ell\left([x, y]^{-1}\right)\right)\right)+\ell(e)
\end{aligned}
$$

for all integers $m, k$, where the final term $\ell(e)$ is used when $m=k=0$, but can also be added in the remaining cases since it is non-negative. We conclude that

$$
f\left(\left(Y_{1}+\cdots+Y_{2 n}\right)\left(1,-\frac{1}{2}\right)\right) \leqslant A\left|Y_{1}+\cdots+Y_{2 n}\right|+\ell(e)
$$

where $A$ is a quantity independent of $n$; more explicitly, one can take

$$
A:=\max \left(\ell(x), \ell\left(x^{-1}\right)\right)+\frac{1}{2} \max \left(\ell([x, y]), \ell\left([x, y]^{-1}\right)\right) .
$$

Taking expectations, since the random variable $Y_{1}+\cdots+Y_{2 n}$ has mean zero and variance $2 n$, we see from the Cauchy-Schwarz inequality or Jensen's inequality that

$$
\mathbf{E}\left|Y_{1}+\cdots+Y_{2 n}\right| \leqslant\left(\mathbf{E}\left|Y_{1}+\cdots+Y_{2 n}\right|^{2}\right)^{1 / 2}=\sqrt{2 n}
$$

and hence

$$
f(0, n) \leqslant A \sqrt{2 n}+\ell(e)+4 c n .
$$

But from (2.7), if $n$ is a power of 2 then we have

$$
\ell([x, y]) \leqslant \frac{f(0, n)}{n}+c .
$$

Combining these two bounds and sending $n \rightarrow \infty$, we obtain Proposition 2.1.

Remark 2.14. One can deduce a 'local' version of Proposition 2.1 as follows: notice that the constant $c$ can be described in terms of $\ell$ from (2.3), to yield

$$
\ell([x, y]) \leqslant 5 \sup _{z \in G}\left(2 \ell(z)-\ell\left(z^{2}\right)\right)
$$

for any group $G$ and function $\ell: G \rightarrow \mathbb{R}$ for which this supremum exists, and any $x, y \in G$. (Both sides are zero when $G$ is a Banach space and $\ell$ is the norm, so equality is obtained in that case.) It is also enough to consider the supremum over the subgroup of $G$ generated by $x$ and $y$ without loss of generality, which may lead to a better bound on $\ell([x, y])$ than taking 
the supremum over all of $G$. Notice also that the constant $c$ must be nonnegative, from (2.3) and (2.2) with $x=y=e$ :

$$
c \geqslant 2 \ell(e)-\ell\left(e^{2}\right)=\ell(e) \geqslant \ell\left(e^{2}\right)-\ell(e)=0 .
$$

In fact, this reasoning and our results imply that the only way to get $c=0$ on the right-hand side of (2.4) is when $\ell$ arises from pulling back the norm of a Banach space $\mathbb{B}$ along a group homomorphism $G \rightarrow \mathbb{B}$, or equivalently along a group homomorphism from the torsion-free abelianization of $G$ to $\mathbb{B}$.

\section{Completing the Proof of Theorem 1.2}

With Proposition 2.1 in hand, it is not difficult to conclude the proof of Theorem 1.2. Suppose that $G$ is a group with a homogeneous semilength function $\ell: G \rightarrow[0,+\infty)$. Applying Proposition 2.1 with $c=$ 0 , we conclude that $\ell([x, y])=0$ for all $x, y \in G$, thus by the triangle inequality $\ell$ vanishes on the commutator subgroup $[G, G]$, and therefore factors through the abelianization $G_{\mathrm{ab}}:=G /[G, G]$ of $G$. Observe that this already establishes part of one implication of Corollary 1.4. Factoring out by $[G, G]$ like this, we may now assume without loss of generality that $G$ is abelian. To reflect this, we now use additive notation for $G$, thus for instance $\ell(n x)=|n| \ell(x)$ for each $x \in G$ and $n \in \mathbb{Z}$, and one can also view $G$ as a module over the integers $\mathbb{Z}$.

At this point we repeat the arguments in [10, Theorem B], which treated the case when $G$ was separable, though it turns out that this separability hypothesis is unnecessary.

If $x$ is a torsion element of $G$, i.e. $n x=0$ for some $n$, then the homogeneity condition forces $\ell(x)=0$. Thus $\ell$ vanishes on the torsion subgroup of $G$; factoring out by this subgroup, we may thus assume without loss of generality that $G$ is not only abelian, but is also torsion-free.

We can view $G$ as a subgroup of the $\mathbb{Q}$-vector space $G \otimes_{\mathbb{Z}} \mathbb{Q}$, the elements of which can be formally expressed as $\frac{1}{n} x$ for natural numbers $n$ and elements $x \in G$ (with two such expressions $\frac{1}{n} x, \frac{1}{m} y$ identified if and only if $m x=n y$, and the $\mathbb{Q}$-vector space operations defined in the obvious fashion); the fact that this is well defined as a $\mathbb{Q}$-vector space follows from the hypotheses that $G$ is abelian and torsion-free. We can then define the map \|\|$_{\mathbb{Q}}: G \otimes_{\mathbb{Z}} \mathbb{Q} \rightarrow$ $[0,+\infty)$ by setting

$$
\left\|\frac{1}{n} x\right\|_{\mathbb{Q}}:=\frac{1}{n} \ell(x)
$$

for any $x \in G$ and natural number $n$; the linear growth condition ensures that \|\|$_{\mathbb{Q}}$ is well-defined. It is not difficult to verify that \|\|$_{\mathbb{Q}}$ is indeed a seminorm over the $\mathbb{Q}$-vector space $G \otimes_{\mathbb{Z}} \mathbb{Q}$.

The norm \|\|$_{\mathbb{Q}}$ on $G \otimes_{\mathbb{Z}} \mathbb{Q}$ gives a metric $d(x, y)=\|x-y\|_{\mathbb{Q}}$. Consider the metric completion $\mathbb{B}$ of $G \otimes_{\mathbb{Z}} \mathbb{Q}$ with this metric. It is easy to see that the $\mathbb{Q}$-vector space structure on $G \otimes_{\mathbb{Z}} \mathbb{Q}$ extends to an $\mathbb{R}$-vector space structure on $\mathbb{B}$, and the norm \|\|$_{\mathbb{Q}}$ on $G \otimes_{\mathbb{Z}} \mathbb{Q}$ extends to a norm \|\|$_{\mathbb{R}}$ on $\mathbb{B}$. As $\mathbb{B}$ is complete by construction, it is a Banach space. The inclusion of $G$ in $G \otimes_{\mathbb{Z}} \mathbb{Q}$ gives a homomorphism $\phi: G \rightarrow \mathbb{B}$ as required. 
This concludes the proof of Theorem 1.2. Since the homomorphism $\phi$ : $G \rightarrow \mathbb{B}$ can only be injective for abelian torsion-free $G$, we obtain the "only if" portion of Corollary 1.4. Conversely, if a group $G$ is abelian and torsionfree, by the above constructions it embeds into a real vector space $\mathbb{B}:=$ $G \otimes_{\mathbb{Z}} \mathbb{R}$; now by Zorn's lemma $\mathbb{B}$ has a norm (e.g., consider the $\ell^{1}$ norm with respect to a Hamel basis of $\mathbb{B}$ ), which restricts to the desired homogeneous length function on $G$. We remark that $G \otimes_{\mathbb{Z}} \mathbb{R}$ is the construction of the smallest, 'enveloping' vector space containing a copy of the abelian, torsionfree group $G$.

Remark 3.1. The above arguments also show that homogeneous pseudolength functions on $G$ are in bijection with seminorms on the real vector space $G_{\mathrm{ab}, 0} \otimes_{\mathbb{Z}} \mathbb{R}$, where $G_{\mathrm{ab}, 0}$ denotes the torsion-free abelianization of $G$.

\section{Further REMARKS AND RESUlts}

If we weaken any of several conditions in Corollary 1.4, then examples of non-abelian structures with generalized length functions do, in fact, often exist. However, the generality of Proposition 2.1 allows us to obtain nontrivial information in some of these cases. Here we mention several such cases and discuss other related problems.

4.1. Monoids and embeddings. Our first weakening is to replace 'groups' by the more primitive structures 'monoids' or 'semigroups'. In this case, Robert Young (private communication) described to us non-abelian monoids with homogeneous, bi-invariant length functions: consider the free monoid FMon $(X)$ on any alphabet $X$ of size at least 2 , with the edit distance $d(v, w)$ between strings $v, w \in \mathrm{FMon}(X)$ being the least number of single generator insertions and deletions to get from $v$ to $w$. The triangle inequality and positivity are easily verified, while homogeneity of the corresponding length function $\ell(x):=d(e, x)$ is trivial. Moreover, the metric $d(\cdot, \cdot)$ turns out to be bi-invariant:

$$
d(g x h, g y h)=d(x, y) \text { for all } g, h, x, y \in \operatorname{FMon}(X) .
$$

This specializes to left- and right-invariance upon taking $g \in X$ and $h=e$, or $h \in X$ and $g=e$, respectively.

Note moreover that $\mathrm{FMon}(X)$ embeds into the free group $\mathrm{FGp}(X)$ generated by $X$ and $X^{-1}$, where $X^{-1}$ is the collection of symbols defined to be inverses of elements of $X$. In particular, $F M o n(X)$ is cancellative. While this trivially addresses the embeddability issue, notice that a more refined version of embeddability fails. Namely, by our main theorem, $\mathrm{FMon}(X)$ does not embed into any group in the category $\mathcal{C}_{\text {bi-inv, hom }}$ with cancellative semigroups with homogeneous bi-invariant metrics as objects and isometric semigroup maps as morphisms. Thus, one may reasonably ask what is a sufficiently small category in which the embeddability works. The following proposition shows that we just need to drop homogeneity.

Proposition 4.1. Let $\mathcal{C}_{\mathrm{bi}-\mathrm{inv}}$ denote the category whose objects are cancellative semigroups with bi-invariant metrics, and morphisms are isometric semigroup maps. Then $\mathrm{FMon}(X)$ embeds isometrically into $\mathrm{FGp}(X)$ in $\mathcal{C}_{\text {bi-inv }}$. 
Proof. From above, $\mathrm{FMon}(X)$ is an object of $\mathcal{C}_{\mathrm{bi} \text {-inv }}$; denote the metric by $d_{F M}$. One can check that $d_{F M}\left(w, w^{\prime}\right)$ equals the difference between $\ell(w)+\ell\left(w^{\prime}\right)$ and twice the length of the longest common (possibly noncontiguous) substring in $w, w^{\prime}$; here, $\ell$ denotes the length of a word in the alphabet $X$.

We next claim $\operatorname{FGp}(X)$ is also an object of $\mathcal{C}_{\text {bi-inv }}$. Namely, for a word $w=x_{1} x_{2} \cdots x_{m}$ in the free group, we consider non-crossing matchings in $w$, i.e., sets $M$ of pairs of letters in $\{1,2, \ldots m\}$ such that the following hold.

- If $(i, j) \in M$, then $i<j$ and $x_{j}=x_{i}^{-1}$.

- If $(i, j),(k, l) \in M$, then either $(i, j)=(k, l)$ or $i, j, k, l$ are distinct.

- If $i<k<j<l$ and $(i, j) \in M$, then $(k, l) \notin M$.

Given a matching $M$ as above, consider the set $U=U(M)$ of indices $k, 1 \leqslant k \leqslant m$ which are not part of a pair in $M$. Define the deficiency of the matching $M$ as the cardinality of the set $U(M)$, and define the length $\ell_{w c}(w)$ of the word $w$ as the infimum of the deficiency over all noncrossing matchings in $w$ (the subscript in $\ell_{w c}$ stands for Watson-Crick). This length was previously studied in [6], including checking that it is welldefined on all of $\operatorname{FGp}(X)$; moreover, $\ell_{w c}(w)$ equals the smallest number of conjugates of elements in $X \sqcup X^{-1}$ whose product is $w$. Now define $d_{F G}\left(w, w^{\prime}\right):=\ell_{w c}\left(w^{-1} w^{\prime}\right)$. It is easy to see that $\ell_{w c}$ is a conjugacy invariant length function.

We claim that $d_{F G} \equiv d_{F M}$ on $\mathrm{FMon}(X)$, which proves the result. It is easy to show that if two words in $\mathrm{FMon}(X)$ differ by a single insertion or deletion, then their distance in $\operatorname{FGp}(X)$ is at most one, hence exactly one. In the other direction, we claim that a non-crossing matching on $w^{-1} w^{\prime}$, with $w$ and $w^{\prime}$ containing only positive generators (in $X$ ), is just a 'rainbow', i.e. nested arches with one end in $w^{-1}$ and the other in $w^{\prime}$. But then $d_{F G}\left(w, w^{\prime}\right)$ equals $\ell(w)+\ell\left(w^{\prime}\right)$ minus twice the length of a common substring, which is maximal by the minimality of the deficiency. Hence $d_{F G}\left(w, w^{\prime}\right)=d_{F M}\left(w, w^{\prime}\right)$, completing the proof.

Note that given weights $\ell(a)$ and $\ell(b)$, there is a natural weighted version $\ell_{w c ; a, b}$ where the letters of $U$ as above are taken with these weights (symmetrically under inversion). This corresponds to the weighted edit distance, with different costs for editing different letters.

4.2. Quasimorphisms and commutator lengths. We now investigate potential applications of Proposition 2.1 with $c>0$. A quasi-morphism on a group $G$ is a map $f: G \rightarrow \mathbb{R}$ whose defect is bounded,

$$
D(f):=\sup _{x, y \in G}|f(x y)-f(x)-f(y)|<+\infty .
$$

Every quasi-morphism induces a pseudo-length function (in particular semilength function) by setting

$$
\ell(x):=|f(x)|+D(f),
$$

where we can take $c=2 D(f)$ as a bound on the homogeneity defect. In this case, Proposition 2.1 makes a rather trivial statement: a homogeneous quasi-morphism is bounded on commutators,

$$
|f([x, y])| \leqslant 10 D(f) .
$$


In fact, as observed in [1, Lemme 1.1], for homogeneous quasi-morphisms one can improve the constant from 10 to 3 , and a quasi-morphism can always be homogenized by replacing it by $\lim _{n \rightarrow \infty} f\left(x^{n}\right) / n$ [1, p. 135], which differs from the original $f$ by at most $D(f)$.

Nevertheless, quasi-morphisms can be utilized to construct interesting pseudo-length functions, for example satisfying homogeneity on specific commutators. The following quasi-morphism is due to Brooks [5, Section 2]. For a given word $w$ in the free group $\mathbf{F}_{2}$, written in reduced form, let $f_{w}: \mathbf{F}_{2} \rightarrow \mathbb{R}$ be the function which assigns to every other $g \in \mathbf{F}_{2}$, also written in reduced form, the maximum number of times such that $w$ occurs in $g$ without overlaps, minus the analogous number of times that $w^{-1}$ can maximally occur in $g$. Since $f_{w}\left(w^{n}\right)=n f_{w}(w)$, using (4.2) results in a pseudo-length function that grows linearly on the powers of $w$. For example with $w$ being the commutator of the generators of $\mathbf{F}_{2}$, we see that although the pseudolength function must be bounded on commutators by Proposition 2.1, it can nevertheless grow linearly on the powers of a fixed commutator.

Thus, there exist examples of quasi-homogeneous semi-length functions on free groups that are not induced by norms. Nevertheless, we will now see that for a large class of groups, including amenable groups and $G=S L(n, \mathbb{Z})$ for $n \geqslant 3$, even all quasi-homogeneous semi-length functions are induced by norms on Banach spaces. Further, the bound from Proposition 2.1 even in the case of free groups is sharper than that obtained without using homogeneity.

Recall that the commutator length $\operatorname{cl}(g)$ of a word in $[G, G]$ is the length $k$ of the shortest expression $g=\left[a_{1}, b_{1}\right] \cdot\left[a_{2}, b_{2}\right] \cdots\left[a_{k}, b_{k}\right]$ of $g$ as a product of commutators. The stable commutator length is defined as $\lim _{n \rightarrow \infty} \operatorname{cl}\left(g^{n}\right) / n$, where the limit exists by sub-additivity of the function $n \mapsto \operatorname{cl}\left(g^{n}\right)$.

Then Proposition 2.1, together with $\ell(e) \leqslant c$ and (2.7) for $n$ a power of two,

$$
\ell(x) \leqslant \frac{\ell\left(x^{n}\right)}{n}+\log _{2}(n) c,
$$

easily imply the following estimates:

Proposition 4.3. Let $\ell$ and $c$ be as in Proposition 2.1. Then for $x \in[G, G]$, $\ell(x) \leqslant(5 \operatorname{cl}(x)+1) c$ and $\ell(x) \leqslant(5 \operatorname{scl}(x)+1) c$.

We say two semi-length functions $\ell_{1}, \ell_{2}: G \rightarrow \mathbb{R}$ are equivalent if $\left|\ell_{1}(x)-\ell_{2}(x)\right|$ is bounded in $x \in G$.

For a group $G$ which is perfect and so that the stable commutator length vanishes on $G=[G, G]$, for example $S L(n, \mathbb{Z})$ for $n \geqslant 3$, it is immediate that any homogeneous semi-length function is bounded, and hence equivalent to the trivial semi-length function $\ell(g) \equiv 0$.

More generally, for groups $G$ for which the stable commutator length vanishes on $[G, G]$, we can deduce an analogue of Theorem 1.2. Note that there are several interesting examples of such groups, including solvable groups, and more generally, amenable groups.

Theorem 4.4. Let $G$ be a group such that the stable commutator length vanishes on $[G, G]$ and assume $\ell: G \rightarrow \mathbb{R}$ satisfies (2.2) and (2.3). Then there exist a real Banach space $\mathbb{B}=(\mathbb{B},\|\|)$ and a group homomorphism $\phi: G \rightarrow \mathbb{B}$ such that $\ell$ is equivalent to $x \mapsto\|\phi(x)\|$. 
Remark 4.5. As in Remark 2.5, we can replace (2.2) by the a priori weaker condition that $\ell(x y) \leqslant \ell(x)+\ell(y)+k$ for all $x, y \in G$ with $k$ fixed.

Proof. Let ab : $G \rightarrow G_{\mathrm{ab}}=G /[G, G]$ be the abelianization homomorphism. We first construct a homogeneous semi-length function $\bar{\ell}$ on $G_{\mathrm{ab}}$ so that $\ell$ is equivalent to $\bar{\ell} \circ \mathrm{ab}$. Let $\eta: G_{\mathrm{ab}} \rightarrow G$ be a section of ab and let $\bar{\ell}_{0}(x):=\ell(\eta(x))+c$. We show that $\bar{\ell}_{0}$ is a semi-length function. The required $\bar{\ell}$ will be obtained by homogenizing $\bar{\ell}_{0}$.

By Proposition 4.3, as the stable commutator length vanishes on $[G, G]$, it follows that for $x, y \in G$, if $\mathrm{ab}(x)=\mathrm{ab}(y)$, then $|\ell(x)-\ell(y)| \leqslant c$. Now, for $\alpha, \beta \in G_{\mathrm{ab}}, \operatorname{ab}(\eta(\alpha \beta))=\mathrm{ab}(\eta(\alpha) \eta(\beta))$, hence

$$
\mid \ell(\eta(\alpha \beta))-\ell(\eta(\alpha) \eta(\beta))) \mid \leqslant c .
$$

This together with the triangle inequality (2.2) gives

$$
\bar{\ell}_{0}(\alpha \beta) \leqslant \bar{\ell}_{0}(\alpha)+\bar{\ell}_{0}(\beta)+c,
$$

while using (2.3) instead gives the required lower bound for $\bar{\ell}_{0}\left(\alpha^{2}\right)$.

Next, for $x \in G$, as $\operatorname{ab}(\eta(\operatorname{ab}(x)))=\operatorname{ab}(x)$, we have $\left|\ell(x)-\left(\bar{\ell}_{0} \circ \mathrm{ab}\right)(x)\right| \leqslant c$. Thus $\ell$ is equivalent to $\bar{\ell}_{0} \circ$ ab.

Since $(\alpha \beta)^{n}=\alpha^{n} \beta^{n}$ in $G_{\mathrm{ab}}$, we also have $\bar{\ell}_{0}\left((\alpha \beta)^{n}\right) \leqslant \bar{\ell}_{0}\left(\alpha^{n}\right)+\bar{\ell}_{0}\left(\beta^{n}\right)+c$. We deduce that the homogenization $\bar{\ell}$ of $\bar{\ell}_{0}$ is a semi-length function on $G_{\mathrm{ab}}$, which is equivalent to $\bar{\ell}_{0}$ due to the bounds (2.6) and (2.7), applied to $\bar{\ell}_{0}$. Therefore also $\ell$ is equivalent to $\bar{\ell} \circ$ ab on $G$.

The claim now follows upon applying Theorem 1.2 to $\left(G_{\mathrm{ab}}, \bar{\ell}\right)$ and taking $\phi$ to be the composition $G \rightarrow G_{\mathrm{ab}} \rightarrow \mathbb{B}$.

The following examples of length functions on the free group show that some hypotheses are needed to get bounds as strong as those of the Theorem (naturally the stable commutator length does not vanish in the free group). For example, consider the word $\left[a^{k}, b^{m}\right]$ in the free group $\mathbf{F}_{2}$, generated by $a$ and $b$, for some integers $k$ and $m$.

- The norm of such an element with respect to the word metric is $2(|k|+|m|)$.

- If we have a length function $\ell$ which is symmetric and conjugationinvariant, but not necessarily homogeneous, then we have the bound $\ell\left(\left[a^{k}, b^{m}\right]\right) \leqslant 2 \min (|k| \ell(a),|m| \ell(b))$. Furthermore, the $\ell_{w c ; a, b}$ from above are conjugation-invariant length functions for which these inequalities hold with equality.

Further, $\ell\left(\left[a^{k}, b^{m}\right]\right) \geqslant 2 \min (|k| \ell(a),|m| \ell(b))$ as, for any matching $M$ for $w=\left[a^{k}, b^{m}\right]$, if some pair $(i, j)$ corresponds to letters $a$ and $a^{-1}$, then no pair corresponds to letters $b$ and $b^{-1}$ and conversely. Further, it is easy to find a matching for $w$ for which the deficiency is $\min (|k| \ell(a),|m| \ell(b))$. On the other hand, $\ell_{w c ; a, b}$ is not homogeneous; for instance, $\ell([a, b])=2$ and $\ell\left([a, b]^{3}\right)=4$. Similarly, we have $\ell\left(\left[a^{k}, b^{k}\right]\right)=2|k|$ and $\ell\left(\left[a^{k}, b^{k}\right]^{3}\right) \leqslant 4|k|$, which demonstrates that $2 \ell(x)-\ell\left(x^{2}\right)$ is unbounded (as must be the case, according to $(2.15))$.

- On the other hand, the function $\ell_{c y c}$ associating to each word the length of its cyclically reduced form is homogeneous, but not a semilength function. For this we have $\ell_{c y c}\left(\left[a^{k}, b^{m}\right]\right)=2(|k|+|m|)$. 
Observe that all of the bounds on $\ell\left(\left[a^{k}, b^{m}\right]\right)$ here become unbounded as $k, m \rightarrow \infty$. This should be compared with Proposition 2.1, which establishes a bound $\ell\left(\left[a^{k}, b^{m}\right]\right) \leqslant 5 c$ that is uniform in $k$ and $m$ for any function $\ell$ satisfying the hypotheses of that proposition.

4.3. Finite balls in free groups. From Proposition 2.1 and a standard compactness argument, we can establish the following local version of the theorem.

Theorem 4.6. For any $\varepsilon>0$ there exists $R \geqslant 4$ with the following property: if $a, b$ are two elements of a group $G, B_{a, b}(R) \subset G$ is the collection of all words in $a, b, a^{-1}, b^{-1}$ of length at most $R$ (so in particular $B_{a, b}(R)$ contains $[a, b])$, and the map $\ell: B_{a, b}(R) \rightarrow[0,+\infty)$ is a "local semi-length function" which obeys the triangle inequality

$$
\ell(x y) \leqslant \ell(x)+\ell(y)
$$

whenever $x, y, x y \in B_{a, b}(R)$, with equality when $x=y$, then one has

$$
\ell([a, b]) \leqslant \varepsilon(\ell(a)+\ell(b)) .
$$

Proof. By pulling back to the free group $\mathbf{F}_{2}$ generated by $a$ and $b$, we may assume without loss of generality that $G=\mathbf{F}_{2}$. Without loss of generality we may also normalize $\ell(a)+\ell(b)=1$. If the claim failed, then one could find a sequence $R_{n} \rightarrow \infty$ and local pseudo-length functions $\ell_{n}: B_{a, b}\left(R_{n}\right) \rightarrow$ $[0,+\infty)$ such that $\ell_{n}(a)+\ell_{n}(b)=1$, but that $\ell_{n}([a, b]) \geqslant \varepsilon$. By the ArzelaAscoli theorem, we can pass to a subsequence that converges pointwise to a homogeneous pseudo-length function $\ell: G \rightarrow[0,+\infty)$ such that $\ell([a, b]) \geqslant \varepsilon$, which contradicts Proposition 2.1.

Remark 4.8. By carefully refining the arguments in the previous section, choosing $n$ to be various small powers of $R$ instead of sending $n$ to infinity, one can extract an explicit value of $R$ of the form $R=C \varepsilon^{-A}$ for some absolute constants $C, A>0$; we leave the details to the interested reader.

On the other hand, for any finite $R$ one can construct local length functions $\ell: B(0, R) \rightarrow[0,+\infty)$ such that $\ell(x)>0$ for all $x \in B(0, R) \backslash\{e\}$. One construction is as follows. Any two matrices $U_{a}, U_{b} \in S O(3)$ define a representation $x \mapsto U_{x}$ of the free group $\mathbf{F}_{2}$ in the obvious fashion. Every $U_{x}$ is then a rotation around some axis in $\mathbb{R}^{3}$ by some angle $0 \leqslant \theta_{x} \leqslant \pi$ in one of the two directions around that axis; if $U_{a}$ and $U_{b}$ are sufficiently close to the identity, then the angle $\theta_{x}$ is at most $\pi / 2$ for all $x \in B(0, R)$. We set $\ell(x):=\theta_{x}$ for $x \in B(0, R)$. Also, if $U_{a}, U_{b}$ are chosen generically, the representation is faithful, as follows from the dominance of word maps on simple Lie groups such as $S O(3)$, see [2]. Hence $\ell(x)>0$ for any nonidentity $x$. From the triangle inequality for angles we thus have (4.7) whenever $x, y, x y \in B(0, R)$, with equality when $x=y$. Note that as one sends $R \rightarrow \infty$, the local length functions constructed here converge to zero pointwise, so in the limit we do not get any counterexample to the main theorem.

\section{REFERENCES}

[1] C. Bavard, Longueur stable des commutateurs, L'Enseignement Mathématique, 37(12):109-150, 1991. 
[2] A. Borel, On free subgroups of semisimple groups, L'Enseignement Mathématique, 29(1-2):151-164, 1983.

[3] N.G. de Bruijn and P. Erdös, Some linear and some quadratic recursion formulas. II, Indagationes Mathematicae, 55:152-163, 1952.

[4] F. Cabello Sánchez and J.M.F. Castillo, Banach space techniques underpinning a theory for nearly additive mappings, Dissertationes Mathematicae (Rozprawy Matematyczne), 404:1-73, 2002.

[5] K. Fujiwara, Quasi-homomorphisms on mapping class groups, Handbook of Teichmüller theory. Vol. II, 241-269, IRMA Lect. Math. Theor. Phys., 13, Eur. Math. Soc., Zürich, 2009.

[6] S. Gadgil, Watson-Crick pairing, the Heisenberg group and Milnor invariants, Journal of Mathematical Biology 59(1), Article:123, 2009.

[7] Z. Gajda and Z. Kominek, On separation theorems for subadditive and superadditive functionals, Studia Mathematica, 100(1):25-38, 1991.

[8] T. Gowers and M. Nielsen, Massively collaborative mathematics, Nature, 461(7266):879-881, 2009.

[9] A. Khare and B. Rajaratnam, The Hoffmann-Jørgensen inequality in metric semigroups, Annals of Probability, 45(6A):4101-4111, 2017.

[10] A. Khare and B. Rajaratnam, The Khinchin-Kahane inequality and Banach space embeddings for metric groups, preprint (arXiv:1610.03037), 2016.

[11] S. Kobayashi, Hyperbolic Manifolds and Holomorphic Mappings, World Scientific, 1970.

[12] M. Ledoux and M. Talagrand, Probability in Banach Spaces (Isoperimetry and Processes), Ergebnisse der Mathematik und ihrer Grenzgebiete, Springer-Verlag, BerlinNew York, 1991.

[13] J. Milnor, Curvatures of left invariant metrics on lie groups, Advances in Mathematics, 21(3):293-329, 1976.

[14] A. Naor, An introduction to the Ribe program, Japan Journal of Mathematics, 7:167233, 2012.

[15] P. Niemiec, Universal valued Abelian groups, Advances in Mathematics, 235:398-449, 2013.

HTTP: //MICHAELNIELSEN. ORG/POLYMATH1/INDEX. PHP 